\title{
ESPECTROSCOPIA RAMAN DE BAIXA FREQUÊNCIA - THZ-RAMAN
}

\author{
D. M. BERTOLDO ${ }^{1}$ \\ Instituto Federal do Triângulo Mineiro ${ }^{1}$, Universidade de Salzburg ${ }^{1}$ \\ durval@iftm.edu.br ${ }^{1}$
}

Submetido 10/04/2016 - Aceito 28/03/2020

DOI: $10.15628 /$ holos.2020.4396

\section{RESUMO}

Este trabalho aborda a espectroscopia Raman de baixa frequência, nomeada usualmente de terahertz Raman. Aqui são mostrados os benefícios associados a esta versátil técnica de análise estrutural, uma vez que seu emprego, na análise da estrutura química dos materiais, vem ganhando espaço em ritmo acelerado. Também apresentamos alguns recentes e didáticos resultados, bem como as limitações desta técnica.

Palavras-Chave: Espectroscopia Raman. Terahertz Raman. Análise estrutural.

\section{LOW-FREQUENCY RAMAN SPECTROSCOPY - THZ-RAMAN}

\section{ABSTRACT}

This work explains about the low-frequency Raman spectroscopy, called Raman terahertz, displaying the benefits of this versatile technique of structural analysis, since the use this technique for chemistry structural analysis have been increasingly used. Also, here is some recent and didactic data as well as the technique limitations.

Keywords: Raman Spectroscopy, Raman terahertz, structural analysis. 


\section{INTRODUÇÃO}

A análise de materiais tem duas principais vertentes. Uma é a análise química, na qual a espectroscopia Raman tem uma posição de destaque entre as técnicas utilizadas na investigação microscópica da matéria [1]. A outra vertente se dedica à análise da estrutura química das moléculas, incluindo informações sobre estrutura polimérica, orientação cristalina e fônos, por exemplo. A grande vantagem da espectroscopia Raman está no fato de que a luz espalhada e posteriormente analisada carrega consigo informações sobre níveis vibracionais. Estas informações podem fornecer dados sobre a simetria, o ambiente eletrônico e ligações químicas das moléculas, permitindo uma análise quantitativa e qualitativa da matéria [2]. A difração de raios-X (XRD), Fourier Transform infrared (FTIR) e também a espectroscopia de absorção em baixa frequência, $\tilde{\lambda}<200 \mathrm{~cm}^{-1}$, são as técnicas mais utilizadas atualmente. Entretanto, estas últimas

técnicas podem ser destrutivas, demandar pré-tratamento físico ou químico das amostras e ser onerosas [3]. Já a espectroscopia Raman é uma técnica que não agride a integridade física ou química das amostras e, por este motivo, ela pode ser utilizada para estudar sangue, plantas, obras de arte, alimentos, dentre outros, além de não requer pré-tratamento físico ou químico e apresentar baixo custo de operação.

Assim, este trabalho é dedicado principalmente à técnica de espectroscopia Raman de baixa frequência, conhecida também por terahertz Raman (THz-Raman), e tem o objetivo de mostrar os benefícios associados a esta versátil técnica de análise estrutural, uma vez que seu emprego, na análise da estrutura química dos materiais, vem ganhando espaço em ritmo acelerado.

\section{EFEITO RAMAN}

O efeito Raman foi previsto teoricamente pelo físico austríaco Adolf Gustav Stephan Smekal, sendo publicado na revista Naturwissenschaften, em 1923 [4], e foi experimentalmente comprovado em 1928 pelo físico indiano Chandrasekhara Venkata Raman [5].Em seu célebre artigo, Raman explicou o fenômeno do espalhamento inelástico da luz, conhecido hoje como Efeito Raman [6]. Ao analisar o espectro de emissão produzido por espelhamento inelástico, Raman verificou que uma pequena fração da luz espalhada sofria um deslocamento no comprimento de onda, caracterizando um espelhamento inelástico. Em suas pesquisas, ele também verificou que tal deslocamento estava associado à estrutura química das moléculas espalhadoras [7].

A radiação eletromagnética, monocromática, incidente em uma amostra pode sofrer difusão elástica e inelástica. As ondas eletromagnéticas com determinada energia por fóton 
$\left(E_{\mathrm{f}}=h v_{f}\right)$ podem ser absorvidas e difundidas elasticamente, caracterizando o conhecido espalhamento Rayleigh. Neste caso, $(h)$ é a constante de Planck e $(v)$ é a frequência da radiação eletromagnética. Esse último efeito ocorre com grande fração da radiação eletromagnética incidente na amostra, mas uma pequena parte pode ser difundida inelasticamente, caracterizando assim o efeito Raman. No espalhamento Raman, a energia dos fótons difundidos, $(E=h v)$, pode ser $E>E_{\mathrm{f}}$ ou $E<E_{\mathrm{f}}[2]$. A Figura 1 mostra os dois primeiros estados vibracionais de uma molécula $\left(n_{0}\right.$ e $n_{1}$ ), com respectivas energias vibracionais $E_{0}$ e $E_{1}$, onde são apresentados três distintos casos de espalhamentos. Entretanto, nos casos mostrados na figura 1 a) e b), a molécula se encontra inicialmente no estado vibracional fundamental $n_{0}$ com energia vibracional $E_{0}$. Em a), apresenta-se o espalhamento Rayleigh, onde a energia do fóton espalhado é $E=h v_{f}$, igual a energia do fóton incidente, $E_{f}=h v_{f}$. Em b), o fóton é espalhado com energia $E=h v$, menor que a do fóton incidente. Por fim, em c), onde a molécula se encontra no primeiro estado vibracional excitado $n_{1}$, com energia $E_{1}$, o fóton difundido, nesse caso, apresenta energia maior que a do fóton incidente. Nos processos $\mathbf{b}$ ) e c), ocorrem espalhamentos inelásticos, caracterizando o efeito Raman, pois nestes processos existem diferenças entre as energias dos fótons incidentes, $E_{\mathrm{f}}$ com as energias dos fótons espalhados, $E$, ou seja $E \neq E_{\mathrm{f}}$

Portanto, o espalhamento Raman pode produzir fótons com energias menores que as energias dos fótons incidentes, $E=E_{f}-E_{1}$, mas pode também produzir fótons com energias maiores, $E=E_{f}+E_{1}$. Por razões históricas, esses dois processos são denominados Espalhamentos Stokes e anti-Stokes $[2,7]$, respectivamente.

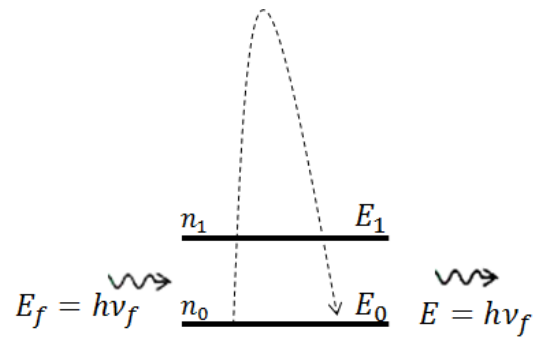

a)

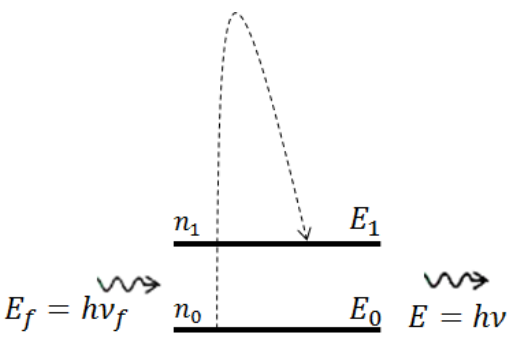

b)

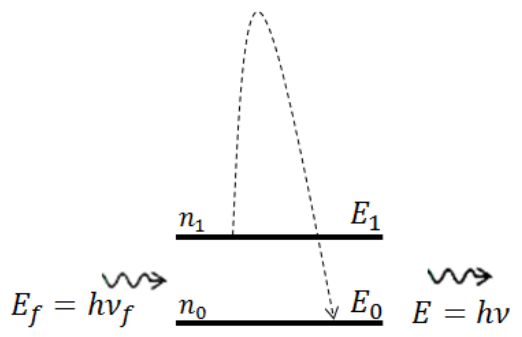

c)

Figura 1: Processo de absorção e espalhamento Raman. Em a), espalhamento Rayleigh ( $\left.E=E_{\mathrm{f}}\right)$; em b), espalhamento Stokes, $\left(E<E_{\mathrm{f}}\right)$; em $\left.\mathrm{c}\right)$, o espalhamento anti-Stokes, $\left(E>E_{\mathrm{f}}\right.$ ).

É válido observar que, no processo mostrado em b), a molécula ganhou energia, pois o fóton espalhado tem energia menor que o fóton incidente. Neste caso, a molécula passa do estado vibracional $n_{0}$ para o estado $n_{1}$, ou seja, sua energia vibracional aumentou de $E_{0}$ para $E_{1}$, ganhando assim $\left(E_{1}-E_{0}\right)$ de energia vibracional. Já no processo mostrado em c), a molécula perdeu energia, pois a mesma se encontrava no estado vibracional $n_{1}$, com energia $E_{1}$, e passou para o estado vibracional $n_{0}$ com energia $E_{0}$. Assim, ela perdeu uma quantidade de energia igual 
$\left(E_{0}-E_{1}\right)$. Portanto, podemos notar que a diferença entre as energias do fóton incidente com a energia do fóton espalhado corresponde à energia de um determinado estado vibracional da molécula, também chamado de modo de vibração molecular. A sutileza deste processo está no fato de que os fótons (luz) adentram na matéria e interagem com a mesma, por meio de processos quânticos, sendo posteriormente espalhados, trazendo consigo informações sobre os estados vibracionais das moléculas, o que, por sua vez, está diretamente relacionado ao ambiente químico molecular. Ou seja, essas informações vêm direto das ligações química, que são as fontes responsáveis pela integridade da matéria. Devido a este motivo, a espectroscopia Raman é tão importante nas análises dos materiais.

Quando se coleta um espectro Raman, são coletadas intensidades dos espalhamentos Raman, geralmente, em função do inverso do comprimento de onda ( $\lambda$ ), analisado pelo instrumento de medida (Espectrômetro ou Espectrofotômetro Raman). O inverso do comprimento de onda é frequentemente denominado deslocamento Raman $(\tilde{\lambda})$, de tal forma que, $\tilde{\lambda}=\frac{1}{\dot{\lambda}}$ Assim, a energia por fóton, $E=h v$, pode ser escrita por $E=h c \tilde{\lambda}$, uma vez que $v=c \tilde{\lambda}$. Dado que (c) é a velocidade da luz no vácuo. Ou seja, a energia associada ao espalhando Raman é diretamente proporcional ao deslocamento Raman ( $\tilde{\lambda}$ ). Um espectro Raman é composto por bandas denominadas bandas Raman, sendo as mesmas associadas a cada específico tipo de processo vibracional dos grupos funcionais de uma molécula. A figura 2 mostra o espectro Raman do nylon $6 / 6$, coletado nas temperaturas de $-100^{\circ} \mathrm{C}$ e $100^{\circ} \mathrm{C}$. Em a), é a parte do espectro entre $900 \mathrm{~cm}^{-1} \mathrm{e}$ $1700 \mathrm{~cm}^{-1}$; em b), entre $2800 \mathrm{~cm}^{-1}$ e $3400 \mathrm{~cm}^{-1}$. Este espectro destaca algumas bandas como a banda centrada em torno de1636 $\mathrm{cm}^{-1}$. Esta banda é associada ao grupo amida I (C=0) [8] e, como pode ser observado na figura 2, ela sofre um deslocamento de frequência para a esquerda, o que significa um aumento da energia de vibração deste grupo funcional. Por outro lado, a banda cristalina associada ao estiramento (C-C-O), centrada em torno de $954 \mathrm{~cm}^{-1}$ [9], sofre um deslocamento para a direita e, portanto, apresenta diminuição da energia vibracional de estiramento do grupo funcional (C-C-O).São comportamentos análogos ao mesmo material, mas que podem ser justificados.
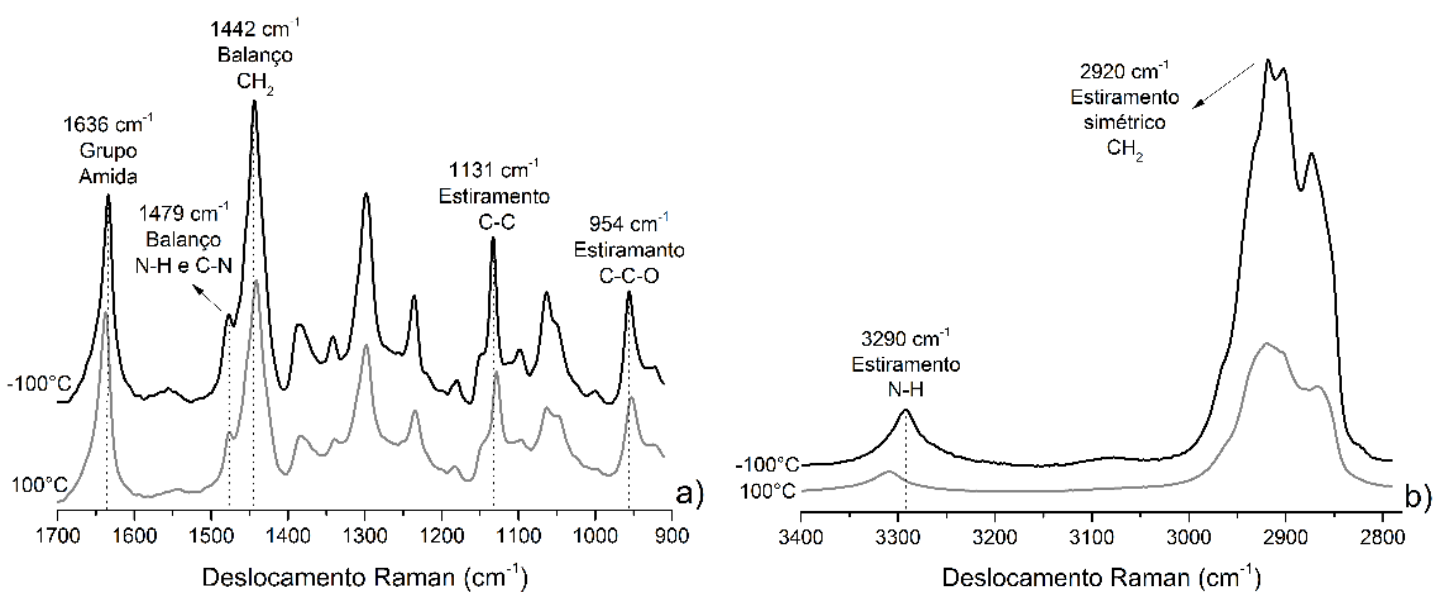

Figura 2: Espectro Raman do nylon $6 / 6$, coletado nas temperaturas de $-100^{\circ} \mathrm{C}$ e $100^{\circ} \mathrm{C}$, com destaque para algumas bandas. a) espectro entre $900 \mathrm{~cm}^{-1} \mathrm{e} 1700 \mathrm{~cm}^{-1}$. b) espectro entre $2800 \mathrm{~cm}^{-1} \mathrm{e} 3400 \mathrm{~cm}^{-1}$. 
O grupo amida I $(\mathrm{C}=\mathrm{O})$ sofre muita influência do ambiente químico devido à interação entre as cadeias moleculares do nylon $6 / 6$, pois existe uma ponte de hidrogênio entre o oxigênio deste grupo com o hidrogênio de uma molécula vizinha do grupo funcional $(\mathrm{N}-\mathrm{H})$, centrado em torno de $3290 \mathrm{~cm}^{-1}$, como mostra a figura 3. Portanto, os grupos amida I (C=O) e $(\mathrm{N}-\mathrm{H})$ sofrem maior influência do ambiente molecular que o grupo funcional ( $C-C-O)$ que está na espinha dorsal do monômero. O mesmo deslocamento para a esquerda pode ser observado na banda associada ao grupo $(\mathrm{N}-\mathrm{H})$, mas é possível observar também que o deslocamento do grupo $(\mathrm{N}-\mathrm{H})$ é mais evidente que o do grupo $(C=O)$. Isto pode ser superficialmente associado ao fato de que o hidrogênio tem menor massa que o oxigênio e, assim, sua frequência de vibração é mais suscetível a variações que o oxigênio.

As mudanças observadas no espectro Raman, neste exemplo, foram em virtude da energia térmica fornecida pela fonte de calor durante o aquecimento da amostra. O aquecimento, com certeza, provocou um aumento do espaço livre entre as cadeias poliméricas [10], o que, por sua vez, conduz a variações nas pontes de hidrogênio, refletidas pela banda em torno de $1636 \mathrm{~cm}^{-1} \mathrm{e}$ $3290 \mathrm{~cm}^{-1}$. Sendo assim, não é difícil perceber a importância da espectroscopia Raman na análise dos materiais.

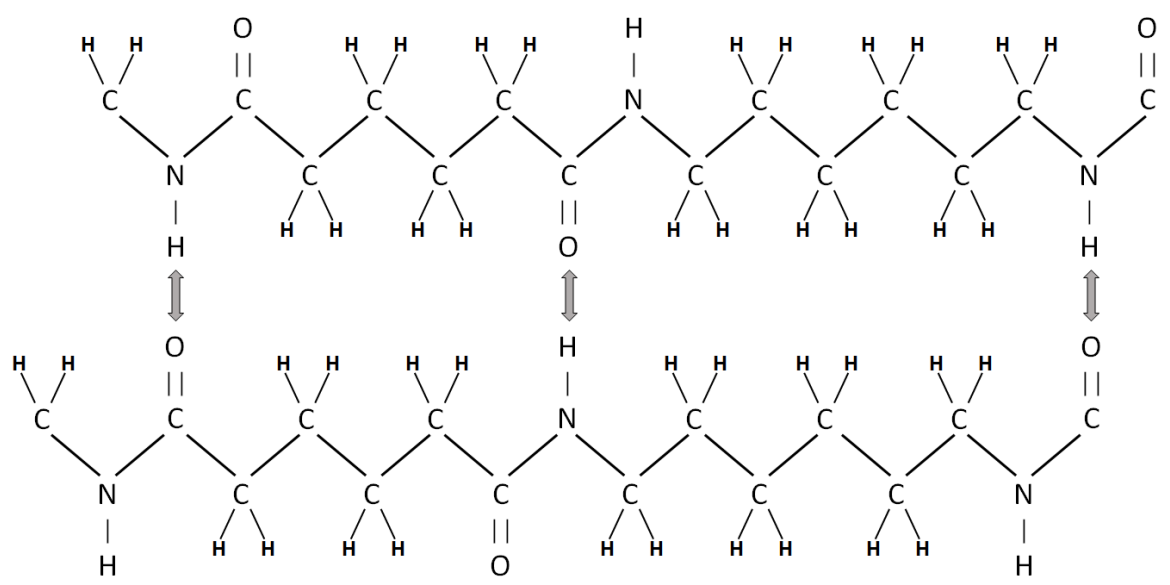

Figura 3:Estrutura planar em zigzag do nylon 6/6, mostrando as ligações de hidrogênio entre duas cadeias vizinhas.

\section{ESPECTROSCOPIA RAMAN TRADICIONAL E ESPECTROSCOPIA RAMAN DE BAIXA FREQUÊNCIA}

A tradicional espectroscopia Raman é associada aos modos de vibrações moleculares, na faixa espectral entre $200 \mathrm{~cm}^{-1} \mathrm{a} 4000 \mathrm{~cm}^{-1}$, de vários tipos de grupos funcionais de uma molécula [7], o que corresponde à faixa espectral associada com o estiramento sub-molecular ou flexões das cadeias moleculares. Esta região espectral é frequentemente chamada de "impressão digital" 
das moléculas, pois é possível, através dela, identificar ou distinguir a composição química de um material. Energias vibracionais com frequências abaixo de $200 \mathrm{~cm}^{-1}$ estão associadas com mudanças estruturais nas moléculas, como deformações estruturais dentro da rede cristalina, por exemplo, e são geralmente difíceis de serem medidas [11].

Portanto, o que difere a tradicional espectroscopia Raman da espectroscopia Raman de baixa frequência, chamada THz-Raman, não são apenas as diferentes faixas espectrais, mas sim o rol de informações que uma pode fornecer em relação a outra. Enquanto a tradicional espectroscopia Raman está associada a informações químicas, aTHz-Raman está associada a informações estruturais. Entretanto, é importante citar que as duas técnicas são altamente complementares, tornando a análise de materiais cada vez mais detalhada e precisa. A figura 4 mostra um espectro vibracional de um material hipotético, onde é possível ver a primeira faixa espectral entre 0 a $200 \mathrm{~cm}^{-1}$, associada à THz-Raman, e a segunda faixa para frequências maiores que $200 \mathrm{~cm}^{-1}$, associada à tradicional espectroscopia Raman.

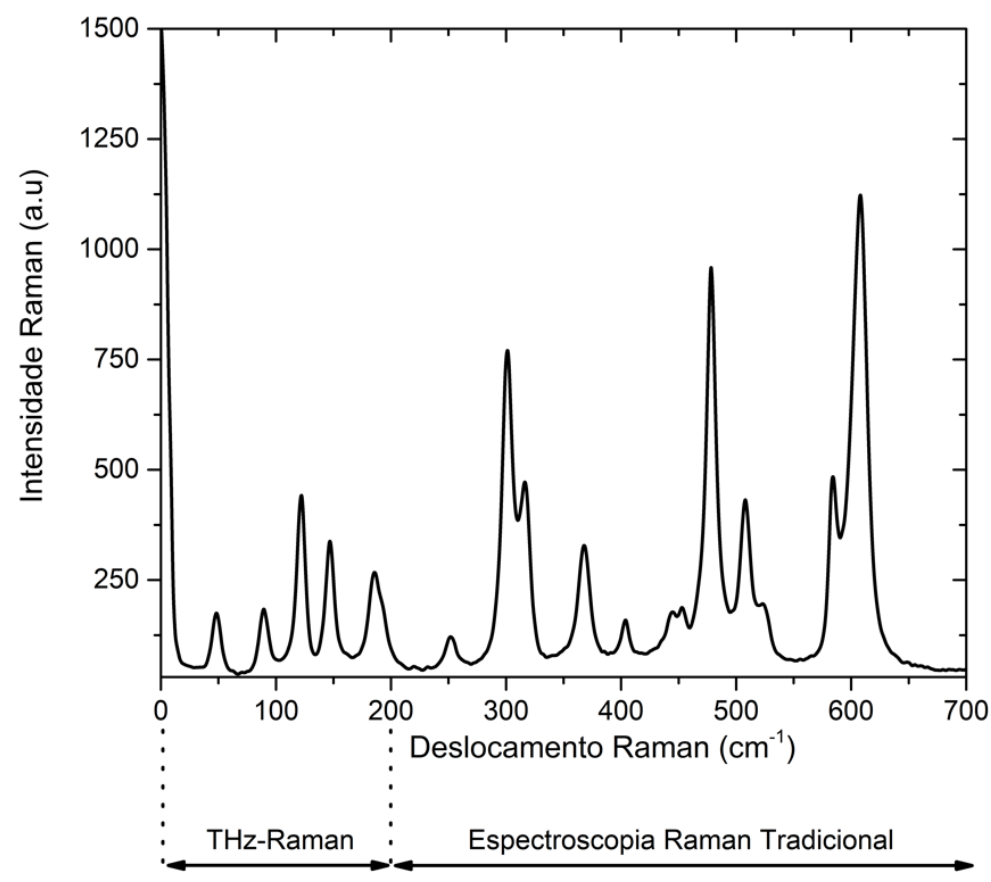

Figura 4: Espectro vibracional de um material hipotético. Primeira faixa espectral $\left(0-200 \mathrm{~cm}^{-1}\right)$, terahertz Raman (THz-Raman), e segunda faixa espectral $\left(>200 \mathrm{~cm}^{-1}\right)$, espectroscopia Raman tradicional.

Na figura 5, são mostradas todas as faixas espectrais Raman, bem como o espalhamento Raman Stokes e anti-Stokes. 


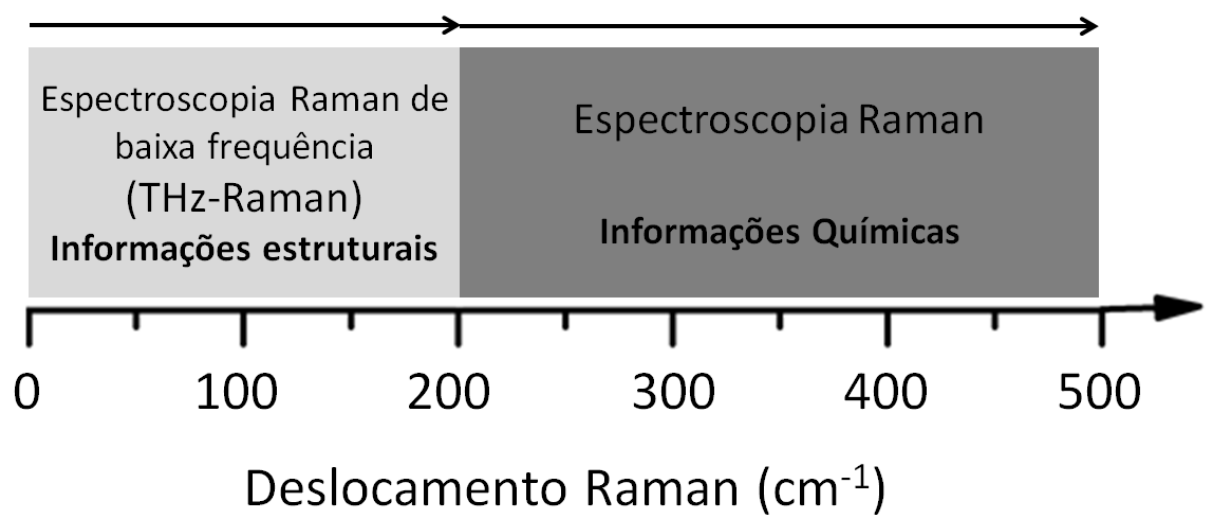

Figura 5: Faixa espectral da espectroscopia terahertz Raman (THz-Raman) entre $0 \mathrm{~cm}^{-1}$ a $200 \mathrm{~cm}^{-1}$ e faixa espectral da espectroscopia Raman tradicional, maior que $200 \mathrm{~cm}^{-1}$.

Contudo, apenas devido a recentes desenvolvimentos na tecnologia, como filtros de corte de banda ultra estreita, é que se tornaram viáveis medições em THz-Raman [12]. Uma das empresas a desenvolver equipamentos para medidas em THz-Raman é a americana Ondax, que já conta com equipamento patenteado, THz-Raman ${ }^{\circledR}$ Raman Spectroscopy Systems, o qual combina medidas em tradicional Raman com o THz-Raman [13].

\section{BENEFÍCIOS, RESULTADOS E LIMITAÇÕES DA THZ-RAMAN.}

Um dos benefícios da THZ-Raman está no fato de que esta técnica requer pouco ou praticamente nenhum pré-tratamento das amostras em estudo, além de os compactos lasers de diodo poderem ser usados como fonte de excitação e os espectros poderem ser adquiridos em poucos segundos devido à grande capacidade de processamento dos computadores atuais.

A espectroscopia THz-Raman tem trafegado entre a investigação de polimorfismo em semicondutores orgânicos [14], ordenamento molecular em polímeros lineares [15] até a caracterização polimorfo de ingredientes farmacêuticos ativos [11] e ainda pode ser usada na análise de materiais explosivos, como petróleo, biomateriais, dentre outros [3]. Na figura 6, há um bom exemplo através do espectro do poli-L-lisine (PLL) nas conformações $\alpha$-Helicoidal e Enrolamento Aleatório. Na figura a seguir, os dois espectros são visivelmente diferentes, demonstrando, deste modo, que a THz-Raman é capaz de caracterizar duas diferentes conformações de um mesmo material polimérico. 


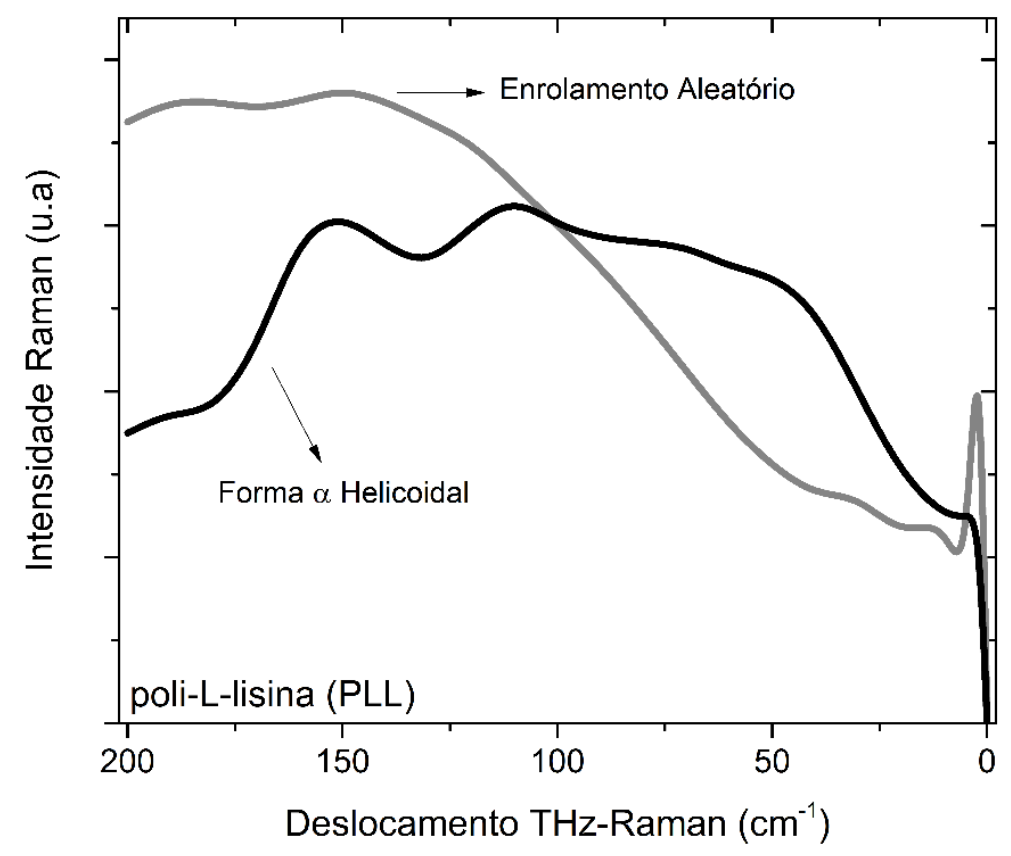

Figura 6: Espectro terahertzRaman (THz-Raman) do poly-L-lysine (PLL) para dois diferentes tipos conformacionais. Conformação $\alpha$-Helicoidal e Enrolamento Aleatório.

Um outro exemplo é mostrado na figura 7, onde Larkin et al [11] mostra que a espectroscopia Raman de baixa frequência é capaz de diferenciar duas formas cristalinas da lactose, neste caso a anidra lactose e a lactose monoidratada.

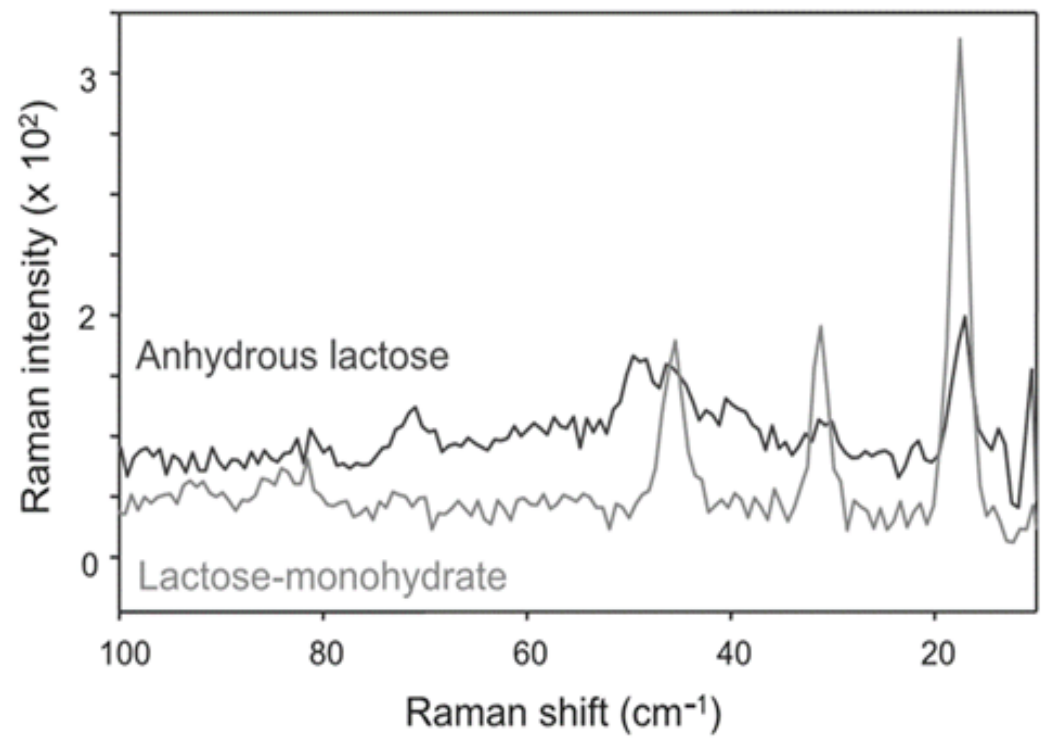

Figura 7:Espectro terahertz Raman (THz-Raman) da lactose monoidratrada e da anidro lactose. Fonte: Larkin et al, "Polymorph Characterization of Active Pharmaceutical Ingredients (APIS) Using Low-Frequency Raman Spectroscopy", Applied Spectroscopy, 68, pag. 774, 2014 [11]. 
O ácido benzoico foi caracterizado por Kutuvantavida e colaboradores [16]. Este ácidoapresenta as formas mostradas na figura $8 \mathrm{em}$ a)ácido benzoico, b) 2-hidroxibenzóico ácido e c) 3-hidroxibenzóico ácido. Faz-se importante verificar, na figura 9, a acentuadadiferença entre os espectros de THz-Raman dessas três formas do ácido. Ou seja, a presença ou a mudança na posição do grupo funcional lateral $(\mathrm{OH})$ leva a óbvias alterações no espectro Raman de baixa frequência, sendo possível caracterizar este material no que tange a sua estrutura química, simplesmente coletando seu espectro THz-Raman.<smiles>O=C(O)c1ccccc1</smiles>

(a)<smiles>O=C(O)c1ccccc1O</smiles>

(b)<smiles>O=C(O)c1cccc(O)c1</smiles>

(c)

Figura 8: Estruturas dos compostos considerados para este estudo; 0 ácido benzoico (a) ácido benzoico, (b) ácido 2hidroxibenzóico, e (c) ácido 3-hidroxibenzóico. Fonte: Kutuvantavida e colaboradores, “Material characterization at low frequencies using THz and Raman spectroscopy", 37th IRMMW-THz, pag. 1-3, 2012 [16].

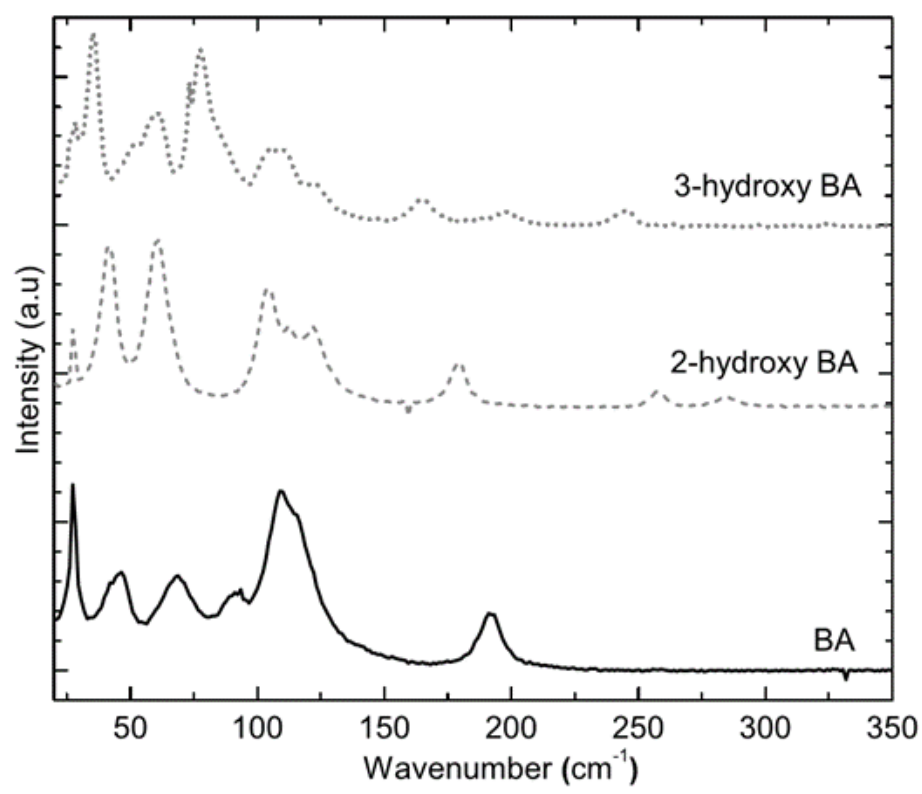

Figure 9: Os espectros de Raman de ácido benzoico, ácido 2-hidroxi, e o ácido 3-hidroxibenzóico em energia corresponde à região de THz-Raman. Fonte: Kutuvantavida e colaboradores, "Material characterization at low frequencies using THz and Raman spectroscopy", 37th IRMMW-THz, pag. 1-3, 2012 [16]. 
Portanto, a espectroscopia Raman de baixa frequência se mostra como uma excelente ferramenta para análise estrutural dos materiais, contendo importantes informações moleculares ou de estrutura cristalina. A espectroscopia Raman de baixa frequência revela uma impressão digital estrutural o que por sua vez é complementada pela espectroscopia Raman tradicional que traz a luz a impressão digital química, o que permite, em simultâneo, a análise estrutural molecular e sua composição química.

Embora a espectroscopia Raman tenha uma posição de destaque na análise de materiais, é sabido que esta técnica tem suas limitações. Por exemplo, nem todos os grupos funcionais são ativos no Raman e ainda existe o problema da fluorescência que pode literalmente esconder as bandas Raman. Estes mesmos problemas são encontrados na espectroscopia Raman de baixa frequência. Entretanto, mesmo com suas limitações a THz-Raman se mostra uma técnica rápida e de baixo custo, apresentando uma enorme capacidade de análise de estados cristalinos e amorfos o que auxiliará na melhor compreensão da estrutura da matéria em futuras pesquisas.

\section{Agradecimentos}

Agradeço à Agência Capes, através do programa Ciências sem Fronteiras, pelo suporte prestado, bem como à Universidade de Salzburg, sua parceira, e ao Instituto Federal do Triângulo Mineiro.

\section{REFERÊNCIAS}

FARIA,D. L. A. d., SANTOS L. G. C., GONÇALVES N. S. (1997). "Uma Demonstração Sobre o Espalhamento Inelástico de Luz: Repetindo o Experimento de Raman," Química Nova, vol. 20, pp. 319-323.

DAS, R. S., AGRAWAL, Y. K. (2011). Raman spectroscopy: Recent advancements, techniques and applecations, Vibrational Spectroscopy, vol. 57, pp. 163-176.

CARRIERE, J., HEYLER,R. (2013). Terahertz Raman: Accessing molecular structure with Raman spectroscopy, Laser Focus World, vol. 49, pp. 33-36.

SMEKALA. (1923). Zur Quantentheorie der Dispersion,Naturwissenschaften, vol. 43, pp. 873875.

RAMAN C. V., KRISHNAN,K. S.(1928). A New Type of Secondary Radiation,"Nature, vol. 121, pp. 501-502.

RAMAN,C. V.,KRISHNAN R. S.(1928). "A Change of Wave-length in Light Scattering," Nature, vol. 121, p. 619. 
JUNIOR,E. B. W.,CROSS J. C. D. a. P. C.. (1980). Molecular Vibrations, New York: Dover Publications INC.

BERTOLDO MENEZES, D. et al. (2018). Introduction to Terahertz Raman spectroscopy, Spectroscopy Letters, vol. 51, pp. 438-445.

MILLER, J. V., BARTICK,E. G. (2001). Forensic Analysis of Single Fibers by Raman Spectroscopy, Appl. Spectrosc, vol. 55, pp. 1729-1732.

VASANTHAN, N., MURTHY, N. S.,BRAY,R. G.. (1998). Investigation of Brill Transition in Nylon 6 and Nylon 6,6 by Infrared Spectroscopy, Macromolecules, vol. 31, pp. 8433-8435.

MENARD, K. P., MENARD, N. R. (2015).Dynamic Mechanical Analysis in the Analysis of Polymers and Rubbers, John Wiley \& Sons, Ltd,

P. LARKIN,J., DABROS,M., SARSFIELD,B., CHAN,E., CARRIERE,J. T.,SMITH, B. C. (2014). Polymorph Characterization of Active Pharmaceutical Ingredients (APIs) Using LowFrequency Raman Spectroscopy,Applied Spectroscopy, vol. 68, pp. 758-776.

CARRIERE,J., HEYLER,R. e SMITH, B. (2013). Polymorph Identification and Analysis Using Ultralow-Frequency Raman Spectroscopy, Spectroscopy, vol. 26, pp. 44-50.

ONDAX (2011). "THz-Raman Spectrosocpy System," Ondax Incorporated,. [Online]. Available: http://www.ondax.com/products/sureblock-xlf-clm-thz-raman-system. [Acesso em 10 março 2016].

P. RANZIERI e et. Al. (2009). "Polymorphism and Phonon Dynamics of $\alpha$-Quaterthiophene," ChemPhysChem, vol. 10, p. 657-663.

PAKHOMOV,P. e. a.. (2011). "Application of the Low Frequency Raman Spectroscopy foStudying Ultra-High Molecular Weight Polyethylenes," Macromolecular Symposia, vol. 305, pp. 63-72.

KUTUVANTAVIDA,Y., WILLIAMS,G. V. M., POGSON,E. M., BHUIYAN,D.,RADHANPURA,K. (2012). Material characterization at low frequencies using $\mathrm{THz}$ and Raman spectroscopy,37th IRMMW-THz: International Conference on Infrared, Millimeter, and Terahertz Wave, pp. 13. 